\title{
Decreased CXCR1 and CXCR2 expression on neutrophils in anti-neutrophil cytoplasmic autoantibody-associated vasculitides potentially increases neutrophil adhesion and impairs migration
}

Nan Hu', Johanna Westra', Abraham Rutgers', Berber Doornbos-Van der Meer', Minke G Huitema', Coen A Stegeman ${ }^{2}$, Wayel H Abdulahad', Simon C Satchell, Peter W Mathieson ${ }^{4}$, Peter Heeringa ${ }^{3}$ and Cees G M Kallenberg ${ }^{1 *}$

\begin{abstract}
Introduction: In anti-neutrophil cytoplasmic autoantibody (ANCA)-associated vasculitides (AAV), persistent inflammation within the vessel wall suggests perturbed neutrophil trafficking leading to accumulation of activated neutrophils in the microvascular compartment. CXCR1 and CXCR2, being major chemokine receptors on neutrophils, are largely responsible for neutrophil recruitment. We speculate that down-regulated expression of CXCR1/2 retains neutrophils within the vessel wall and, consequently, leads to vessel damage.

Methods: Membrane expression of CXCR1/2 on neutrophils was assessed by flow cytometry. Serum levels of interleukin-8 (IL-8), tumor necrosis factor alpha (TNF- $\alpha$ ), angiopoietin 1 and angiopoietin 2 from quiescent and active AAV patients and healthy controls (HC) were quantified by ELISA. Adhesion and transendothelial migration of isolated neutrophils were analyzed using adhesion assays and Transwell systems, respectively.

Results: Expression of CXCR1 and CXCR2 on neutrophils was significantly decreased in AAV patients compared to HC. Levels of IL-8, which, as TNF $\alpha$, dose-dependently down-regulated CXCR1 and CXCR2 expression on neutrophils in vitro, were significantly increased in the serum of patients with active AAV and correlated negatively with CXCR1/CXCR2 expression on neutrophils, even in quiescent patients. Blocking CXCR1 and CXCR2 with repertaxin increased neutrophil adhesion and inhibited migration through a glomerular endothelial cell layer.
\end{abstract}

Conclusions: Expression of CXCR1 and CXCR2 is decreased in AAV, potentially induced by circulating proinflammatory cytokines such as IL-8. Down-regulation of these chemokine receptors could increase neutrophil adhesion and impair its migration through the glomerular endothelium, contributing to neutrophil accumulation and, in concert with ANCA, persistent inflammation within the vessel wall.

\footnotetext{
* Correspondence: c.g.m.kallenberg@reuma.umcg.nl

'Department of Rheumatology and Clinical Immunology, University Medical

Center Groningen, PO Box 30.001, 9700 RB, Groningen, The Netherlands

Full list of author information is available at the end of the article
} 


\section{Introduction}

AAV comprises granulomatosis with polyangiitis (GPA), microscopic polyangiitis (MPA) and Churg Strauss syndrome (CSS), which share a spectrum of clinical manifestations reflecting necrotizing damage to small- and medium-sized vessels [1,2]. A role for neutrophils as effector cells in AAV is supported by a large body of evidence from in vitro and in vivo studies. After being primed by proinflammatory cytokines such as TNF- $\alpha$, neutrophils can be activated by ANCA and release oxygen radicals and proteolytic enzymes, which have been shown to lyse endothelial cells in in vitro co-cultures $[3,4]$. In vivo, neutrophil accumulation in glomeruli has been observed in the early phase of crescentic glomerulonephritis and neutrophil depletion could completely prevent disease development in experimental models [5].

Migration of neutrophils is largely regulated by the concentration gradient of CXC-chemokines which contain a glutamic acid-leucine-arginine $\left(\mathrm{ELR}^{+}\right)$motif and are the most powerful chemoattractants for neutrophils. Interleukin 8 (IL-8) is the most potent member of the CXC family with high affinity for both of its receptors, CXCR1 and CXCR2, which are co-expressed on the membrane of neutrophils. Thus, binding of IL- 8 to CXCR $1 / 2$ is a major element in neutrophil recruitment [6].

Neutrophils function in immune surveillance. Their activation, in terms of degranulation and oxidative burst, arms neutrophils with microbicidal activity, which normally appears only after they have migrated from the circulation and reach inflamed tissues [7]. However, in the pathology of AAV, necrotizing damage within the vessel wall suggests that ANCA-mediated neutrophil activation is already triggered during neutrophil recruitment. This may be caused by impaired neutrophil trafficking retaining activated neutrophils within the microvascular compartment.

Although consensus is still lacking whether ANCA bind to neutrophils in suspension, it has been well demonstrated that membrane bound-proteinase 3 (PR3) on neutrophils is significantly upregulated during neutrophil adhesion, and full neutrophil activation not only requires ANCA-antigen cross-linking but also adherence of neutrophils [8-11]. Here, we hypothesize that IL-8CXCR1/CXCR2-mediated neutrophil recruitment is hampered in AAV, which leads to firm adhesion of neutrophils to the endothelium without further transmigration. Retained neutrophils within the microvascular compartment may be activated by ANCA, which will result in persistent inflammation of the vessel wall. To test this hypothesis, we investigated expression profiles of CXCR1 and CXCR2 on neutrophils in patients with AAV, related expression to levels of chemoattractants, and analyzed effects on transmigration.

\section{Materials and methods}

Patients and healthy controls

For measurement of CXCR1 and CXCR2 expression, 37 patients with quiescent AAV and 5 AAV patients with active disease were recruited from our out-patient clinic. Thirty healthy donors recruited from laboratory personnel were included as a normal control population. Characteristics of patients and controls are summarized in Table 1.

A diagnosis of granulomatosis with polyangiitis (GPA), Churg Strauss syndrome (CSS) or microscopic polyangiitis (MPA) was based on the Chapel Hill definitions [12]. ANCA specificity for PR3 or myeloperoxidase (MPO) was determined by capture ELISA. Patients in remission were without treatment. Treatment of active patients is listed in Table 1 . Disease severity was quantified using the Birmingham Vasculitis Activity Score (BVAS).

Table 1 Characteristics of patients and controls.

\begin{tabular}{|c|c|c|c|c|}
\hline \multirow[t]{3}{*}{ Characteristics } & \multicolumn{4}{|c|}{ Number (\%) or mean (range) } \\
\hline & \multirow{2}{*}{$\begin{array}{l}\mathrm{HC} \\
(n=30)\end{array}$} & \multicolumn{3}{|l|}{ AAV } \\
\hline & & $\begin{array}{l}\text { Remission } \\
(n=37)\end{array}$ & $\begin{array}{l}\text { Active }^{a} \\
(n=5)\end{array}$ & $\begin{array}{l}\text { Active }^{b} \\
(n=18)\end{array}$ \\
\hline Male & $17(57)$ & $25(68)$ & $3(60)$ & $14(78)$ \\
\hline Age (years) & $45(28-64)$ & $57(26-84)$ & $58(47-68)$ & $58(32-81)$ \\
\hline \multicolumn{5}{|l|}{ Diagnosis } \\
\hline GPA & & 26 & 4 & 13 \\
\hline MPA & & 9 & 1 & 5 \\
\hline NCGN & & 2 & 0 & 0 \\
\hline \multicolumn{5}{|l|}{ ANCA-specificity } \\
\hline PR3 & & $24(65)$ & $4(80)$ & $13(72)$ \\
\hline MPO & & $13(35)$ & $1(20)$ & $5(28)$ \\
\hline \multicolumn{5}{|l|}{ Organ involvement } \\
\hline ENT & & 0 & $4(80)$ & $5(28)$ \\
\hline Eye & & 0 & $1(20)$ & $4(22)$ \\
\hline Lung & & 0 & $4(80)$ & $8(44)$ \\
\hline Kidney & & 0 & $2(40)$ & $13(72)$ \\
\hline Skin & & 0 & $1(20)$ & 0 \\
\hline Nervous system & & 0 & $1(20)$ & $2(11)$ \\
\hline BVAS, median (range) & & 0 & $14.8(6-26)$ & $17.5(6-33)$ \\
\hline \multicolumn{5}{|l|}{ Treatment } \\
\hline MMF & & 0 & $1(20)$ & $3(17)$ \\
\hline CYC & & 0 & $4(80)$ & $11(61)$ \\
\hline Prednisolon & & 0 & $4(80)$ & $16(89)$ \\
\hline
\end{tabular}

AAV, ANCA-associated vasculitides; ANCA, anti-neutrophil cytoplasmic autoantibody; BVAS, Birmingham Vasculitis Activity Score; CYC, cyclophosphamide; ENT, ear, nose and throat; GPA, granulomatosis with polyangiitis; $\mathrm{HC}$, healthy control; MMF, mycophenolate mophetil; MPA, microscopic polyangiitis; MPO, myeloperoxidase; NCGN, necrotizing crescentic

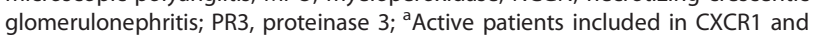
CXCR2 measurement by flow cytometry. ${ }^{b}$ Active patients included in cytokine profiling by ELISA. 
Sera from AAV patients and healthy volunteers were collected for cytokine profiling. For patients in remission, samples were drawn on the same day for CXCR $1 / 2$ measurement and cytokine testing. For patients who were in remission but had suffered from active disease before $(n=18)$, serum samples from active disease were retrieved from a stored serum bank. All sera were stored at $-80^{\circ} \mathrm{C}$ before testing.

All subjects gave their informed consent and the study was approved by the hospital medical ethical committee.

\section{Antibodies used for flow cytometry}

Peridin chlorophyll protein (PerCP)/Cy5.5-conjugated anti-CD14 (HCD14; Biolegend, San Diego, CA, USA) was used to discriminate neutrophils from monocytes in whole blood staining. Phycoerythrin (PE)-conjugated anti-CD181 (CXCR1, 5A12; BD Bioscience, Alphen aan de Rijn, The Netherlands), allophycocyanin (APC)-conjugated anti-CD182 (CXCR2, 6C6; BD Bioscience, Alphen aan de Rijn, The Netherlands) and fluorescein isothiocyanate (FITC)-conjugated anti-CD177 (NB1, MEM166; abCAM, Cambridge, UK) were used to detect membrane expression of CXCR1, CXCR2 and CD177, respectively. Irrelevant antibodies of the same isotype were used as negative controls.

\section{Cell culture}

Human conditionally immortalized glomerular endothelial cells (CiGEnC) [13] were cultured in endothelial growth medium 2-microvascular (EGM2-MV; CambrexLonza, Breda, The Netherlands) containing 5\% FCS and growth factors, without vascular endothelial growth factor (VEGF), as supplement. CiGEnC up to passage 40 were propagated at $33^{\circ} \mathrm{C}$ to keep cells in a proliferative state. Experiments were carried out on cells that had grown into a confluent monolayer and had been incubated for 5 days at $37^{\circ} \mathrm{C}$ in order to acquire a non-proliferative/quiescent phenotype.

\section{Neutrophil isolation and stimulation}

Neutrophils were isolated from peripheral blood according to routine procedures as described previously [14]. Briefly, heparinized venous blood was centrifuged on Lymphoprep (Axis-Shield, Oslo, Norway). Contaminating erythrocytes were lysed with ice-cold ammonium chloride buffer. Afterwards, cells were washed with cold Hanks' balanced salt solution (HBSS) without $\mathrm{Ca}^{2+} / \mathrm{Mg}^{2}$ $+\left(\mathrm{HBSS}^{-/-}\right)$and resuspended in HBSS with $\mathrm{Ca}^{2+} / \mathrm{Mg}^{2+}$ $\left(\mathrm{HBSS}^{+/+}\right.$; GIBCO/Life Technologies, Breda, The Netherlands) to obtain $10^{7}$ cells $/ \mathrm{ml}$.

Where indicated, cells were incubated with serial doses of interleukin-8 (IL-8; R\&D Systems, Minneapolis, MN USA), angiopoietin-1 (ANGPT-1; R\&D Systems, Minneapolis, MN USA), angiopoietin-2 (ANGPT-2;
R\&D Systems, Minneapolis, MN USA), recombinant human tumor necrosis factor- $\alpha$ (rhTNF- $\alpha$; R\&D Systems, Minneapolis, MN USA) or repertaxin (SigmaAldrich, ST. Louis, MO USA) at $37^{\circ} \mathrm{C}$ for 30 minutes. Non-stimulated cells were incubated with control medium under the same condition.

\section{Membrane staining of neutrophils for flow cytometry}

Membrane expression of CXCR1, CXCR2 and CD177 on neutrophils was assessed by flow cytometry. According to the manufacturer's instructions, $100 \mu \mathrm{l}$ of whole blood from patients and controls or $10^{6}$ of purified neutrophils after in vitro stimulation were incubated with antibodies at room temperature, in the dark, for $15 \mathrm{~min}$ utes, followed by fixation and lysing of erythrocytes with $20 \times$ volume of Fluorescence Activated Cell Sorting (FACS) lysing solution (BD Bioscience, San Jose, CA, USA). Fluorescence intensity was measured by FACS Calibur (Becton Dickinson Immunocytometry Systems, Mountain View, CA, USA) and calibrated using CellQuest $^{\mathrm{TM}}$ software (Becton Dickinson). Results were analyzed using the Win-List software package (Verity Software House, Topsham, ME, USA). Mean fluorescence intensity (MFI) of $5.10^{4}$ counted cells was measured in each sample. Neutrophils were gated as forward scatter ${ }^{\text {high }}\left(\mathrm{FSC}^{\text {high }}\right)$-side scatter ${ }^{\text {high }}\left(\mathrm{SSC}^{\text {high }}\right)$ $\mathrm{CD} 14^{\text {low }}$ cells. Expression levels were presented as MFI corrected for nonspecific binding of isotype control antibodies on neutrophils from the same donor.

\section{MPO activity assay}

Numbers of adherent or migrated neutrophils in adhesion or migration assay were quantified by myeloperoxidase (MPO) activity assay [15]. Briefly, cells were lysed with $0.5 \%$ Triton-X-100 at $4{ }^{\circ} \mathrm{C}$ for 20 minutes and acidified by adding $1 \mathrm{M}$ citrate buffer $(\mathrm{pH}=4.2,50 \mu \mathrm{l} / 1 \mathrm{ml}$ of cell lysate) just prior to the reaction. Aliquots $(75 \mu \mathrm{l})$ from each sample were transferred into wells of a flatbottomed 96-well plate. 2.2'-azino-bis-3-ethylbenzothiazoline-6-sulfonic acid (ABTS) at a concentration of $0.05 \%$ in $100 \mathrm{mM}$ citrate with $0.03 \% \mathrm{H}_{2} \mathrm{O}_{2}$ was loaded in an equal volume as substrate for MPO. After the reaction, $\mathrm{OD}_{405}$ was measured using an ELISA plate reader. Numbers of neutrophils in each well were determined as based on standards generated from known concentrations of neutrophils from the same isolation.

\section{Neutrophil adhesion}

Neutrophil adhesion to endothelial monolayers was quantified by adhesion assay as described previously with modifications [16]. CiGEnCs were seeded in 24well plates and allowed to proliferate to confluence. Cell monolayers were carefully washed with $\mathrm{HBSS}^{+/+}$and stimulated with $10 \mathrm{ng} / \mathrm{ml}$ TNF- $\alpha$ at $37^{\circ} \mathrm{C}$ for 4 hours. 
Cells were then washed three times before $0.5 \times 10^{6}$ neutrophils were loaded. Where indicated, neutrophils were pretreated with serial doses of IL-8, TNF- $\alpha$ or repertaxin which is a noncompetitive allosteric inhibitor of CXCR1 and CXCR2 [17]. After incubation of endothelial cells with neutrophils at $37^{\circ} \mathrm{C}$ for $30 \mathrm{~min}$ utes, loosely adherent or non-adherent neutrophils were washed off with $\mathrm{HBSS}^{-1-}$ and the endothelial monolayers plus adherent neutrophils were lysed in $0.5 \%$ Triton-X100. All experiments were run in duplicate. The numbers of neutrophils were quantified, as described above, by MPO activity assay.

\section{Neutrophil migration}

Transwell inserts (Corning, NY, USA), with $3.0 \mu \mathrm{m}$ pores in 12-well plates, were precoated with CiGEnCs. Confluence of the endothelial monolayers was determined by continued monitoring of trans-endothelial electrical resistance (TEER) [13] which plateaued at 20 to $25 \Omega$ on the $5^{\text {th }}$ day after seeding. Histological staining of the membrane showed that the formation of monolayers on inserts required $6 \times 10^{4}$ cells and 5 days of culture. Therefore, all the Transwell inserts were coated with CiGEnCs following this standard procedure. Isolated neutrophils from healthy donors were preincubated with repertaxin, IL- 8 or TNF- $\alpha$, and $1.10^{6}$ cells were loaded in the upper chamber and $10 \mathrm{ng} / \mathrm{ml}$ of IL8 , which showed optimal efficiency as a chemoattactant in titration in preliminary experiments (data not shown), was placed in the lower chamber. Wells without IL-8 in the lower chamber or neutrophils without any treatment in the upper chamber were included as controls. All experiments were run in duplicate. After co-incubation of neutrophils with the inserts at $37^{\circ} \mathrm{C}$ for 2 hours, the plates with inserts within the wells were centrifuged for 5 minutes at $50 \mathrm{~g}$ and $4^{\circ} \mathrm{C}$ to dislodge adherent neutrophils on the lower surface of the inserts. The cells in the lower chamber were collected and quantified by MPO activity assay.

Serum levels of IL-8, TNF- $\alpha$, angiopoietin-1 (ANGPT-1) and angiopoietin-2 (ANGPT-2)

Sera collected from AAV patients in remission $(n=37)$, patients with active disease $(n=18)$ and healthy blood donors $(n=30)$ were used for measurement of IL- 8 , TNF- $\alpha$, ANGPT- 1 and ANGPT-2. IL- 8 and TNF- $\alpha$ levels were measured by ELISA (R\&D Systems, Minneapolis, MN, USA) as described previously [18]. After sample incubation and binding of the biotinylated detecting antibodies, color reaction was performed with streptavidin-poly-HRP (Sanquin, Amsterdam, The Netherlands) and tetramethyl-benzidin (TMB, Roth, Karlsruhe, Germany). Levels of angiopoietin-1 and angiopoietin-2 in sera were measured using ELISA kits
(R\&D Systems, Minneapolis, MN, USA) according to the manufacturer's instructions.

Normal levels of these cytokines in serum were defined as the mean \pm 2 SD of healthy controls.

\section{Statistical analysis}

Results of CXCR1 and CXCR2 expression are presented as medians. Serum levels of IL-8, TNF- $\alpha$, ANGPT- 1 and ANGPT-2 and membrane expression of adhesion molecules are presented as means. Data were analyzed by the Mann-Whitney test and Spearman's rank correlation test using GraphPad Prism 4.03 (GraphPad Software, San Diego, CA, USA). $P$ values less than 0.05 were considered significant.

\section{Results}

Expression of CXCR1 and CXCR2 on the neutrophil membrane is decreased in AAV

We first evaluated expression levels of CXCR1 and CXCR2 on neutrophils in AAV patients (remission, $n=$ 37; active, $n=5$ ) in comparison with healthy blood donors $(n=30)$. Both receptors were highly expressed on the membrane of neutrophils. There was no difference in CXCR1 and CXCR2 expression in patients with different AAV diseases or between patients with MPOor PR3- ANCA specificity (data not shown). Neutrophils from patients in remission (median 1153, range 640 to approximately 1,986$)$ showed comparable expression of CXCR1 as those from $\mathrm{HC}(1,163,790$ to approximately $1,790, P=0.3)$, while neutrophil CXCR1 levels were significantly decreased in patients with active disease (533, 416 to approximately 1,071$)$ compared to either $\mathrm{HC}(P$ $=0.003)$ or patients in remission $(P=0.0023)$ (Figure 1A). CXCR2 expression was significantly down-regulated in AAV patients in remission (453, 201 to approximately 817 ) compared to $\mathrm{HC}(589,290$ to approximately $941, P$ $=0.0004)$ and further decreased in active patients (323, 242 to approximately 564) as compared to $\mathrm{HC}(P=$ 0.0086) (Figure 1B). A significant correlation was observed between the expression of CXCR1 and CXCR2 on neutrophils of AAV patients, regardless of disease activity (Figure $1 \mathrm{C}$, spearman $\mathrm{r}=0.87, P<0.0001$ ).

\section{IL-8 and TNF- $\alpha$ down-regulate CXCR1/CXCR2 from the neutrophil membrane}

IL- 8 and TNF- $\alpha$ are proinflammatory cytokines reported to be elevated in the serum of AAV patients [19-21]. Serum levels of ANGPT-2 have also recently been reported to be increased in AAV and to correlate with disease activity [22]. IL-8 is also produced by endothelial cells stimulated with ANGPT-1 [23]. Therefore, we first tested the influences of these cytokines on CXCR1 and CXCR2 expression on neutrophils of HC. As a result, both IL- 8 and TNF- $\alpha$ down-regulated CXCR1 and 


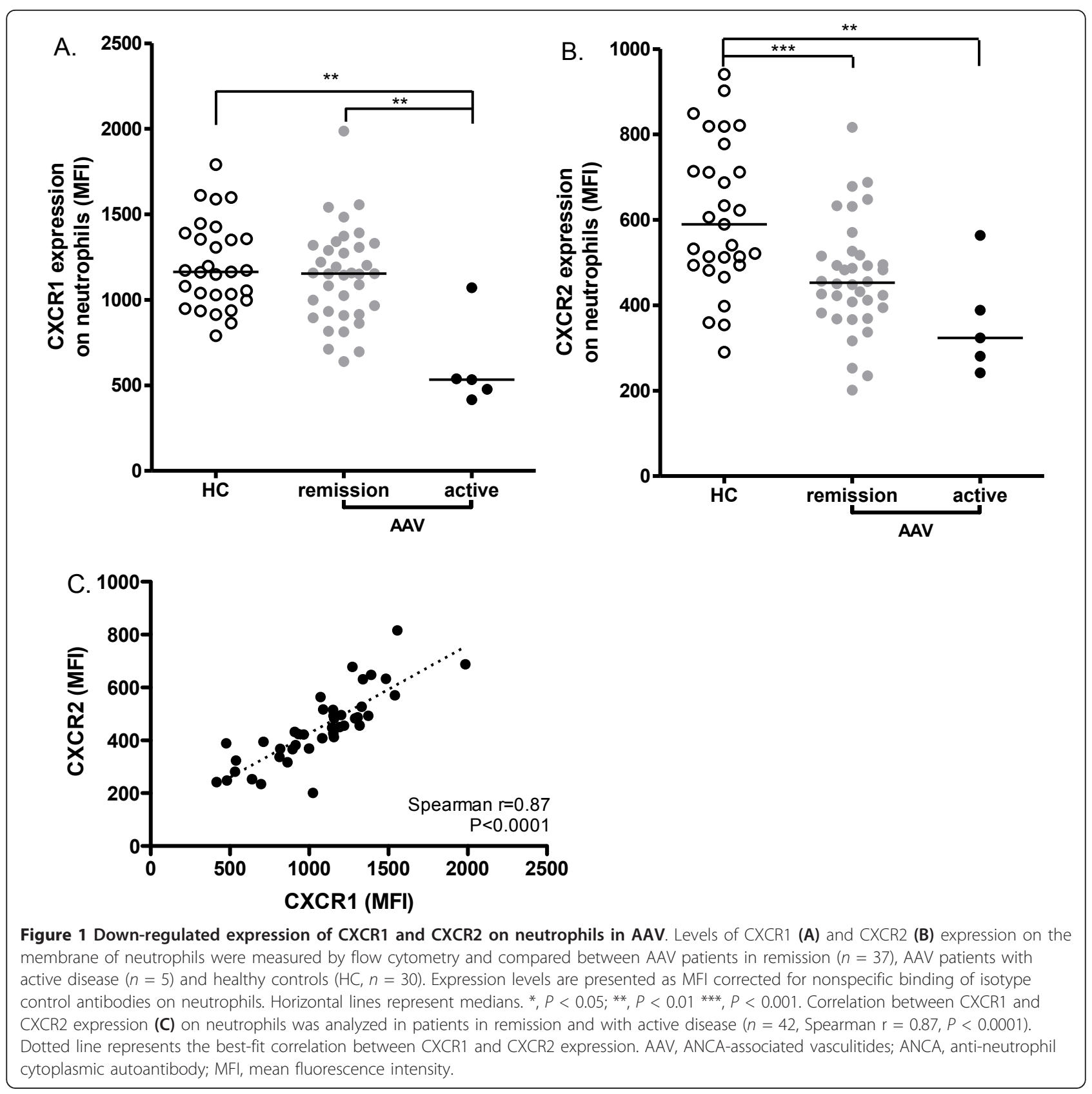

CXCR2 expression on isolated neutrophils in a dosedependent manner. IL-8, at a concentration of $5 \mathrm{ng} / \mathrm{ml}$, reduced CXCR2 levels to $61 \%$ compared to controls incubated with normal medium, while IL-8 down-regulated membrane expression of CXCR1 to $71 \%$ at $20 \mathrm{ng} /$ $\mathrm{ml}$ (Figure 2A left figure). TNF- $\alpha$ significantly reduced CXCR1/CXCR2 expression already at a dose of $1 \mathrm{ng} / \mathrm{ml}$ (Figure 2B left figure). When tested in parallel, CD177 (NB1) expression was slightly increased by IL-8 and significantly up-regulated by TNF- $\alpha$, as reported in earlier studies $[14,24]$. The effects were less obvious when neutrophils of quiescent AAV patients were used. A significant reduction in expression of CXCR1 and CXCR2 was observed only at a concentration of $20 \mathrm{ng} / \mathrm{ml}$ of IL8 (Figure 2A, right figure). Incubation with TNF- $\alpha$ decreased their expression but not to the same extent as seen for $\mathrm{HC}$ neutrophils (Figure 2B, right figure). Possibly AAV neutrophils are already pre-primed in the circulation and, therefore, respond less to in vitro treatment with cytokines. ANGPT-1 and ANGPT-2 did not affect CXCR1/CXCR2 or CD177 expression on isolated neutrophils (Figure 2C and 2D). 

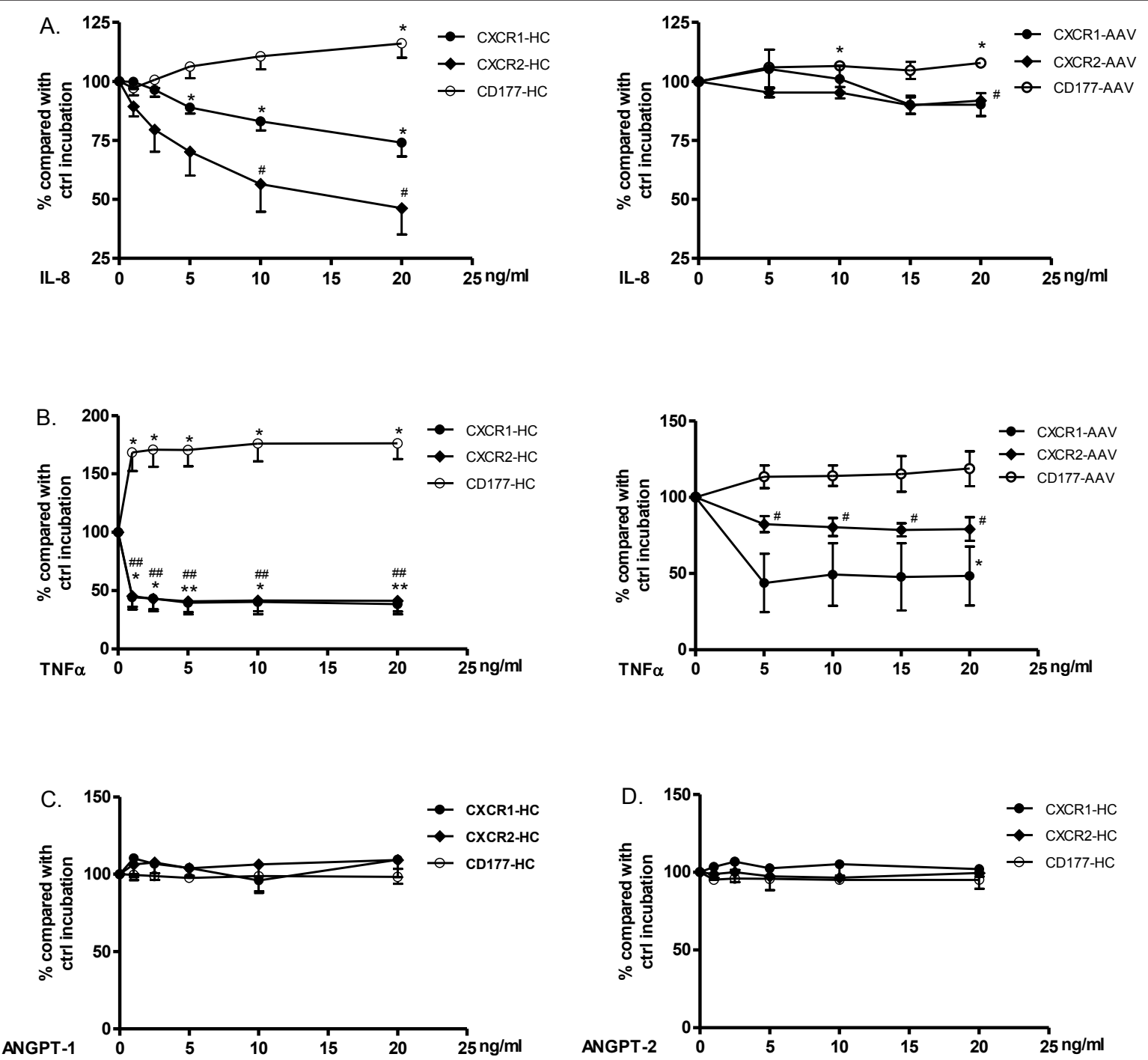

Figure 2 Down-regulation of CXCR1 and CXCR2 on neutrophils by IL-8 and TNF $\alpha$. Isolated neutrophils from HC were pre-incubated with serial doses of IL-8 (A, left figure), TNF $\alpha$ (B, left figure), ANGPT-1 (C) or ANGPT-2 (D). Isolated neutrophils from AAV patients were pre-incubated with serial doses of IL-8 (A, right figure) and TNF $\alpha$ (B, right figure). Membrane expression of CXCR1 (black dots) and CXCR2 (black diamonds) was measured by flow cytometry. Expression of CD177 (open dots) was measured in parallel as control for stimulation. Results are presented as percentages compared to control incubation with normal medium (ctrl). CXCR1 and CXCR2 are equally down-regulated by TNF $\alpha$ (overlapping graphs in panel B). Results are the mean ( \pm SD) of three experiments, ${ }^{*}, P<0.05 ;{ }^{* *}, P<0.01$ (CXCR1 and CD177). \#, $P<0.01$ (CXCR2). AAV ANCA-associated vasculitides; ANCA, anti-neutrophil cytoplasmic autoantibody; ANGPT, angiopoietin; IL; interleukin; TNF, tumor necrosis factor.

Serum levels of IL-8, TNF- $\alpha$, ANGPT-1 and ANGPT-2 in AAV

Levels of the cytokines mentioned above, which may have direct or indirect effects on CXCR1/CXCR2 expression, were evaluated in our patient cohort in comparison with $\mathrm{HC}$.

Defining the normal range as mean \pm 2 SD of the IL-8 concentration in 30 healthy controls, 5 out of 37 (14\%) patients in remission and 10 out of 18 (56\%) active patients showed increased IL-8 levels. Levels of IL-8 were not significantly different between patients with quiescent AAV (median $2.81 \mathrm{pg} / \mathrm{ml}$, range 0.0 to approximately $19.64 \mathrm{pg} / \mathrm{ml})$ and healthy controls $(2.30$ $\mathrm{pg} / \mathrm{ml}, 0$ to approximately $15.43 \mathrm{pg} / \mathrm{ml}$ ). However, a negative correlation was present between CXCR1/ CXCR2 expression on neutrophils and levels of IL-8 in patients in remission (Figure 3 ). A negative correlation was not observed between IL- 8 and CXCR $1 / 2$ in active AAV, probably due to the small sample size. Nevertheless, in concert with further decreased CXCR $1 / 2$ expression, IL-8 levels increased remarkably during active disease $(8.67 \mathrm{pg} / \mathrm{ml}, 3.96$ to approximately $51.55 \mathrm{pg} / \mathrm{ml})$, and were significantly higher than levels in $\mathrm{HC}$ and patients in remission (Figure 4A). 


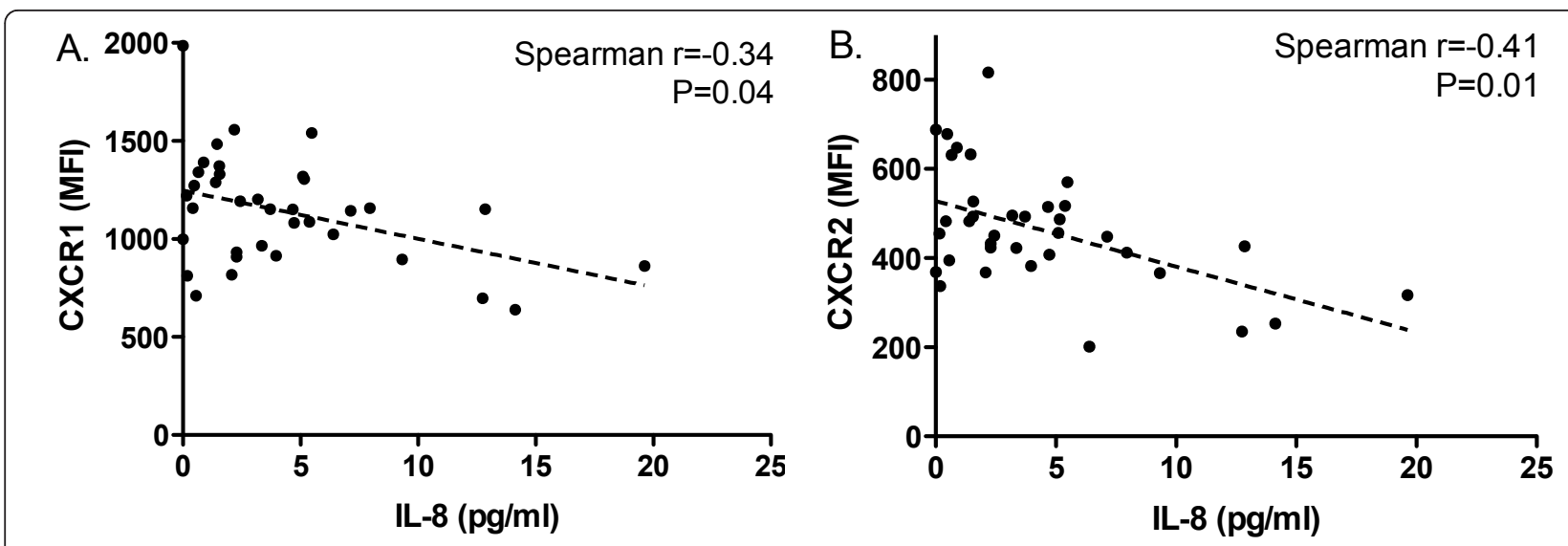

Figure 3 CXCR1/CXCR2 expression correlates with IL-8 levels in quiescent AAV. Expression of CXCR1, CXCR2 and levels of IL-8 were measured in parallel in patients in remission $(n=37)$. Membrane expression of CXCR1 and CXCR2 is presented as MFI corrected for nonspecific binding of isotype control antibodies on neutrophils. IL-8 levels are presented as concentration in serum. A. Correlation between CXCR1 expression on neutrophils and serum levels of IL-8 (Spearman $r=-0.34, P=0.04$ ). B. Correlation between CXCR2 expression on neutrophils and serum levels of IL-8 (Spearman $r=-0.41, P=0.01$ ). Dotted lines represent the best-fit correlation between expression of CXCR1/2 and IL-8 levels. AAV, ANCA-associated vasculitides; ANCA, anti-neutrophil cytoplasmic autoantibody; IL, interleukin.

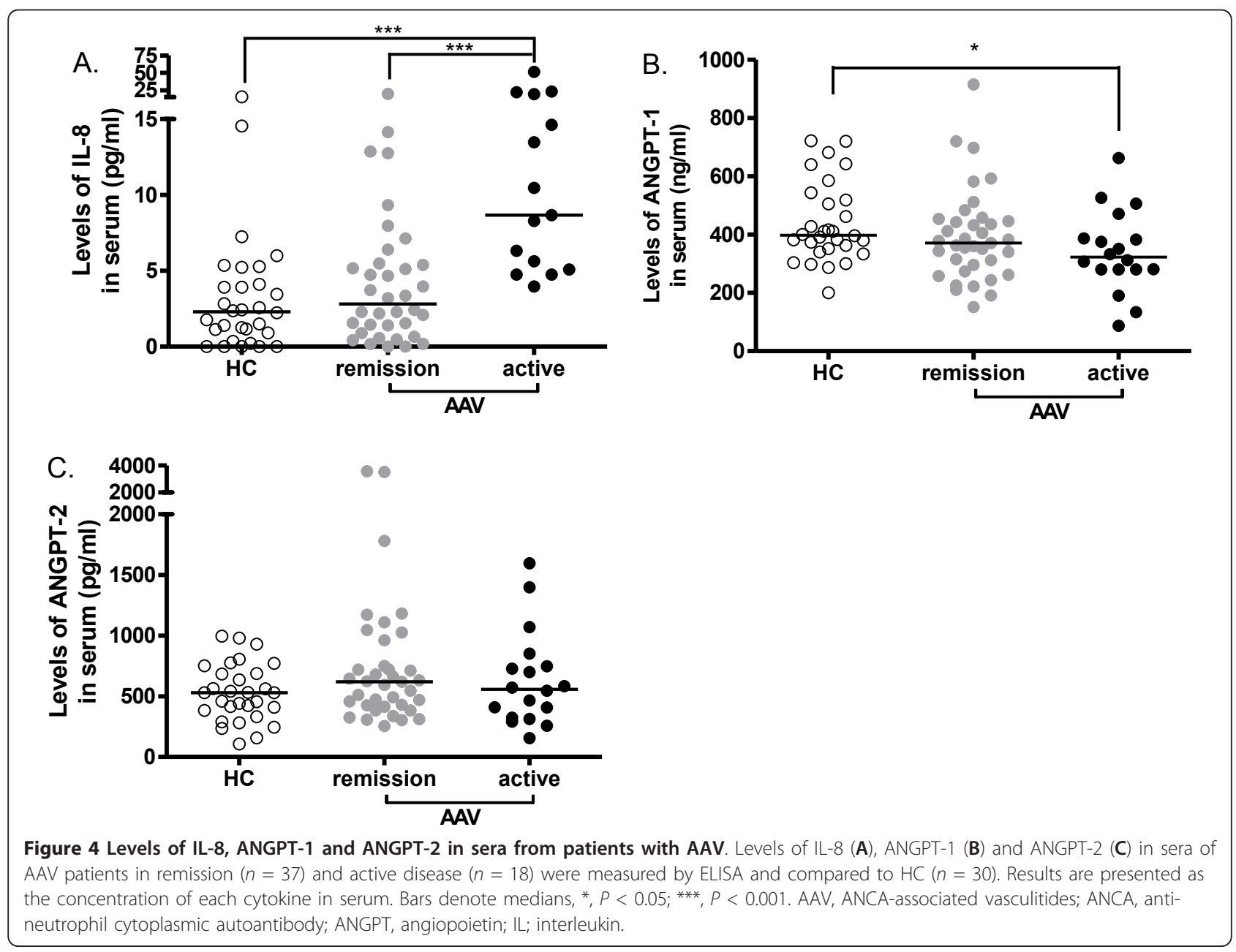


As for levels of ANGPT-1, no significant difference was observed between $\mathrm{HC}$ and patients in remission. The median level of ANGPT-1 in active patients was lower than in HC (Figure 4B). Levels of ANGPT-2 were not increased in patients in remission nor in patients with active disease (Figure 4C). TNF- $\alpha$ was not detectable in sera of either $\mathrm{HC}$ or patient groups.

\section{Neutrophil recruitment after CXCR1/CXCR2 blockade}

To further clarify possible consequences of CXCR1 and CXCR2 down-regulation on neutrophils, we investigated neutrophil adhesion and transmigration through a glomerular endothelial monolayer. Neutrophils were pretreated with repertaxin, mimicking decreased efficiency of IL- 8 receptors, or stimulated with IL-8/TNF- $\alpha$, both of which lead to decline of CXCR1 and CXCR2 expression.

Glomerular endothelial cell layers were stimulated with $10 \mathrm{ng} / \mathrm{ml}$ of TNF- $\alpha$ for 4 hours prior to the adhesion or migration assay to mimic activated glomerular endothelial cells as occur in AAV. Preincubation with repertaxin inhibited neutrophil migration in a dosedependent way. Migration was reduced to $76 \%$ by 100 $\mathrm{nM}$ of repertaxin, significantly lower than control incubated neutrophils. IL- 8 preincubation reduced the number of migrated neutrophils to 39\%. Although incubation with TNF- $\alpha$ resulted in a significantly reduced expression of CXCR1/CXCR2 on the membrane of neutrophils, migration was not significantly influenced, and numbers of migrated neutrophils were comparable with control incubation (Figure 5B).

Neutrophils preincubated with $2 \mathrm{ng} / \mathrm{ml}$ TNF- $\alpha$ showed enhanced adhesion to glomerular endothelium compared to control incubation. IL-8 (up to $20 \mathrm{ng} / \mathrm{ml}$ ), however, did not show a remarkable effect on neutrophil adhesion. Blocking CXCR1 and CXCR2 with repertaxin also increased the number of adherent neutrophils dosedependently, from $135 \%$ with $10 \mathrm{nM}$ of repertaxin up to more than $300 \%$ at $0.5 \mu \mathrm{M}$ as compared to cells without stimulation (Figure 5A).

\section{Discussion}

This is the first study, to our knowledge, reporting decreased expression of CXCR1 and CXCR2 on circulating neutrophils in AAV, potentially induced by proinflammatory cytokines such as IL-8. Functional deficiency of CXCR1/CXCR2, induced by specific inhibition of these receptors with repertaxin, increased neutrophil adhesion and impaired migration through a glomerular endothelium monolayer. Down-regulation of CXCR1 and CXCR2 may retain activated neutrophils within the vessel wall, allowing interaction with circulating ANCA, so leading to vascular damage in situ. These findings highlight a chemokine-receptor-based mechanism of inflammation confined within the vessel wall and would further explain the role of neutrophils in the pathogenesis of AAV.

CXCR1 and CXCR2 are not stably expressed on the neutrophil membrane. During chemotaxis, ligandmediated internalization and recycling of these chemokine receptors is proposed as a self-limiting mechanism allowing neutrophils, to retain in situ by desensitization to their ligands[25]. However, this physiological process is probably abused in AAV, and down-regulated CXCR1/CXCR2 expression may desensitize circulating neutrophils to chemotactic signals before they migrate
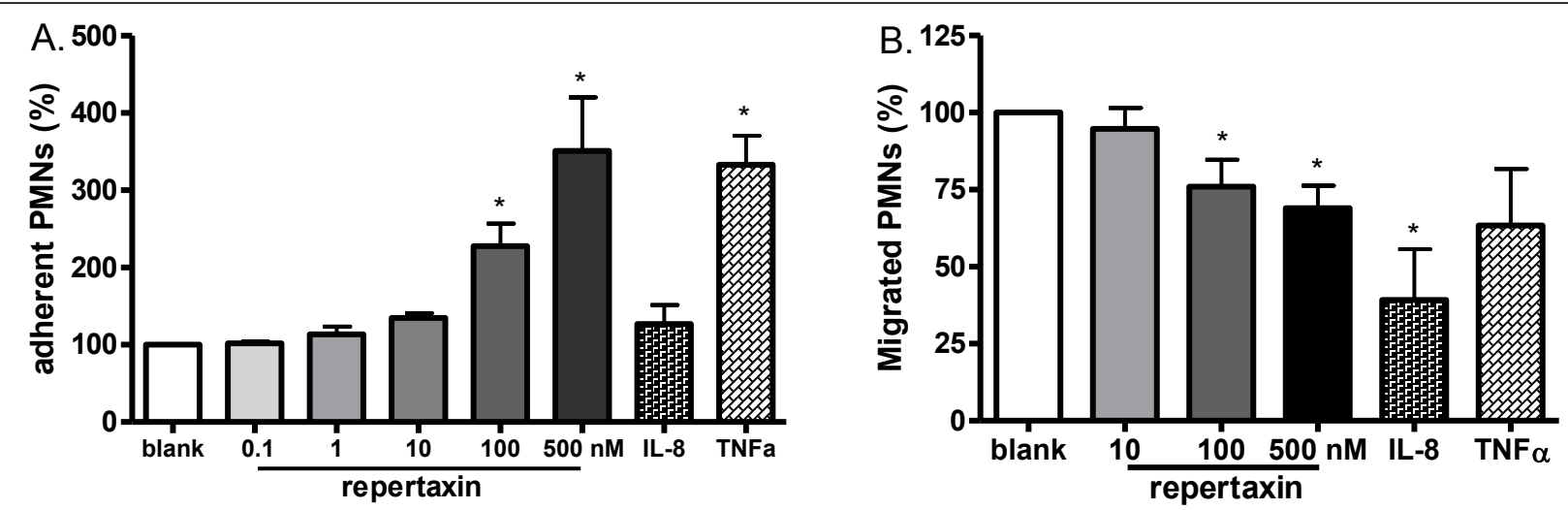

Figure 5 CXCR1/CXCR2 blockade enhances neutrophil adhesion and inhibits neutrophil transmigration through an endothelial cell monolayer. Isolated neutrophils from HC were pre-incubated with serial doses of repertaxin, IL-8 (10 ng/ml) or TNF $\alpha$ (2 $\mathrm{ng} / \mathrm{ml})$ before being loaded onto a glomerular endothelial monolayer or a transwell insert. After co-incubation of pretreated neutrophils with glomerular endothelial cells for 30 minutes for the adhesion assay $(\mathbf{A}, n=5)$ or with transwell inserts coated with endothelial cells for 2 hours $(\mathbf{B}, n=6)$, the number of adherent or migrated neutrophils were quantified by MPO activity assay. Results are presented as the percentages of adherent or migrated neutrophils after stimulation compared to control incubation. ${ }^{*}, P<0.05$. HC, healthy control; IL; interleukin; MPO, myeloperoxidase; TNF, tumor necrosis factor. 
into the tissue resulting in accumulation of neutrophils within the vessel wall.

Our in vitro experiments confirmed this hypothesis by showing significantly increased adhesion and decreased migration of neutrophils through glomerular endothelium after CXCR1 and CXCR2 blockade. This is in line with our previous observation that, in a model of MPOANCA-associated vasculitis, repertaxin treated mice were not protected from hematuria, albuminuria or glomerular crescent formation. Although some neutrophil migration through the endothelial monolayer in our in vitro experiments can not completely be ruled out, we observed a larger number of neutrophils accumulating in glomeruli in comparison to untreated mice [26]. Besides, CXCR1/CXCR2-knockout mice showed a deficiency in neutrophil migration and bacterial clearance in a urinary tract infection model, and decline in CXCR1/ CXCR2 expression may worsen outcome of infections due to impaired neutrophil recruitment [27-31].

Notably, down-regulation of CXCR1 and CXCR2 from the neutrophil surface may not be specific for AAV. As IL- 8 is a potential inducer of decreased CXCR $1 / 2$ expression, increased levels of circulating IL- 8 in other clinical conditions may also result in a similar decline of CXCR1/2 expression. In addition, our results may reflect a state of neutrophil activation mediated by IL- 8 or other proinflammatory cytokines, such as TNF $\alpha$, as has been described in AAV by Muller Kobold et al. previously [32]. In the presence of ANCA, however, preactivated neutrophils lacking CXCR1/2 expression can be confined in the microvasculature and fully activated by ANCA.

The role of CXCR1 and CXCR2 in neutrophil adhesion has not been clearly delineated. Blocking of IL-8receptors showed an inhibitory effect on IL-8-induced neutrophil adhesion in an early study [33]. The underlying mechanisms are not fully elucidated so far. A recent study conducted by Cohen-Hillel et al. showed that migratory desensitization triggers over-activation of focal adhesion kinase (FAK), which leads to increased neutrophil adhesion [34]. The latter mechanism is likely operative in AAV, where neutrophils are migratory desensitized under continuous exposure to low-dose IL8 [20]. Besides, in our experiments, neutrophils pretreated with repertaxin did show significantly increased adhesion on an activated CiGEnC monolayer. As endothelial cells might also be actively involved in neutrophil-endothelial interaction during inflammation, this adhesive effect may also depend on endothelial phenotypes and microenvironment, explaining why Coelho et al. observed short-term suppression of neutrophil adhesion to synovial endothelium after repertaxin treatment in a mouse model of arthritis [35]. Glomerular endothelial cells (ECs) have unique features and differ from synovial ECs in many ways, such as expression profile of adhesion molecules [36]. Neutrophil recruitment in an in vitro model of rheumatoid arthritis only occurs in cocultures of ECs with synovial fibroblasts but not with control tissues [37], suggesting that immune regulators in the microenvironment also play an important role in this process. We are aware of the fact that the cells used in our study were glomerular endothelial cells that were immortalized, and therefore seem artificial. However these cells were shown to have retained morphological features of early-passage primary culture glomerular ECs and they expressed EC adhesion molecules and other specific features [13].

Since CXCR1 and CXCR2 are internalized upon ligation and IL- 8 has been shown to be significantly elevated in AAV patients [20], we speculate that IL-8 is one of the factors inducing decreased expression of these receptors. Indeed, we found that CXCR1 and CXCR2 expression was remarkably lower in patients with active AAV than in HC. Decreased expression was accompanied by significantly elevated serum levels of IL-8 in active AAV. Moreover, a negative correlation was observed between CXCR1/CXCR2 expression and serum IL-8 levels in patients with quiescent disease. As an indirect evidence of this relationship, a negative effect of serum IL-8 on neutrophil recruitment has been demonstrated in earlier studies which was supposed to be caused by down-regulation of IL- 8 receptors $[38,39]$. Cockwell et al. proposed a similar scenario in AAV by showing that ANCA-activated neutrophils produce IL-8 and that neutrophils accumulate locally in the glomerular capillary loops but poorly penetrate into IL-8 enriched tissues as observed in renal biopsies of AAV patients [40]. From the data of the present study, a relationship is suggested between circulating IL-8 levels, decrease in CXCR1/CXCR2 expression and neutrophil accumulation in the microvasculature in AAV.

Circulating IL-8 can be produced by ANCA-activated neutrophils and endothelial cells stimulated by IL-1, TNF- $\alpha$ or PR3-ANCA [40-42]. Although circulating IL8 levels were not significantly elevated in patients during remission, it should be noted that a low-level of immune activation is present during quiescent disease as well, including minor increases in IL-8 levels [43].

CXCR1 and CXCR2 expression can also be regulated, via enzymatic cleavage via metalloproteinases [44], by other immunomodulators such as TNF- $\alpha$ which have been detected at inflammatory sites in AAV and prime neutrophils for ANCA-induced activation [20,45-47]. Technical limitations to measure TNF $\alpha$ in serum could have hampered the revelation of the relationship between TNF $\alpha$ levels and CXCR $1 / 2$ expression. As shown in the current study, TNF- $\alpha$, up to a concentration of $20 \mathrm{ng} / \mathrm{ml}$, was more efficient than IL-8 in down- 
regulating CXCR1/CXCR2 in vitro. Therefore, although TNF- $\alpha$ could not be detected in sera, there is still the possibility that increased levels of TNF- $\alpha$, especially during active disease, lead to a decrease in CXCR1 and CXCR2 expression.

It needs to be mentioned that the number of patients with active AAV included in the current study was relatively small, and so does not allow firm conclusions as to the correlation between neutrophil CXCR1 and CXCR2 expression and disease activity. Such a correlation is, however, suggested by the further decrease of CXCR1 and CXCR2 expression that we observed during active disease. Furthermore, immunohistochemical studies are needed to show lack of CXCR1 and CXCR2 expression on neutrophils recruited to the vascular wall in AAV. Such data could further strengthen the role of CXCR1 and CXCR2 down-regulation in AAV.

\section{Conclusions}

Expression of CXCR1 and CXCR2 on neutrophils is down-regulated in AAV patients, potentially induced by circulating IL-8. Decreased expression of these chemokine receptors may enhance neutrophil adhesion and impede neutrophil migration through activated glomerular endothelium, which may account for neutrophil accumulation and persistent ANCA-induced inflammation in the vessel wall.

\begin{abstract}
Abbreviations
AAV: ANCA-associated vasculitides; ABTS: 2.2'-azino-bis-3-

ethylbenzothiazoline-6-sulfonic acid; ANCA: anti-neutrophil cytoplasmic autoantibody; ANGPT: angiopoietin; APC: allophycocyanin; BVAS: Birmingham Vasculitis Activity Score; CiGEnC: conditionally immortalized glomerular endothelial cell; CSS: Churg Strauss syndrome; CYC: cyclophosphamide; EC: endothelial cell; FCS: fetal calf serum; FITC: fluorescein isothiocyanate; GPA: granulomatosis with polyangiitis; HBSS: Hanks' balanced salt solution; HC: healthy control; IL: interleukin; MFI: mean fluorescence intensity; MMF: mecophenolate mophetil; MPA: microscopic polyangiitis; MPO: myeloperoxidase; PE: phycoerythrin; PR3: proteinase 3; TEER: trans-endothelial electrical resistance; TMB: tetramethyl-benzidin; TNF: tumor necrosis factor; VEGF: vascular endothelial growth factor.
\end{abstract}

\section{Author details}

'Department of Rheumatology and Clinical Immunology, University Medical Center Groningen, PO Box 30.001, 9700 RB, Groningen, The Netherlands. ${ }^{2}$ Department of Nephrology, University Medical Center Groningen, PO Box 30.001, 9700 RB, Groningen, The Netherlands. ${ }^{3}$ Department of Pathology and Medical Biology, University Medical Center Groningen, PO Box 30.001, 9700 RB, Groningen, The Netherlands. ${ }^{4}$ Academic Renal Unit, University of Bristol, Second Floor, Learning and Research, Southmead Hospital, Bristol, BS10 5NB, UK.

\section{Authors' contributions}

$\mathrm{NH}$ made substantial contributions to conception and design of this study, acquisition, analysis and interpretation of data and drafted the manuscript; JW and PH were involved in the study design, data interpretation and revised the manuscript critically; $\mathrm{WHA}, \mathrm{MGH}$ and $\mathrm{BD}$ contributed in the data acquisition; AR and CAS collected patient materials from out-patient clinic; PWM and SCS kindly provided the human glomerular endothelial cell line for this research; CGMK participated in the study design and data analysis and interpretation and revised the manuscript carefully and critically. All authors read and approved the final manuscript.

\section{Competing interests}

The authors declare that they have no competing interests.

Received: 6 June 2011 Revised: 18 October 2011

Accepted: 8 December 2011 Published: 8 December 2011

\section{References}

1. Jennette JC, Falk RJ: Small-vessel vasculitis. N Engl J Med 1997, 337:1512-1523.

2. Kallenberg CG, Heeringa P, Stegeman CA: Mechanisms of disease: pathogenesis and treatment of ANCA-associated vasculitides. Nat Clin Pract Rheumatol 2006, 2:661-670.

3. Falk RJ, Terrell RS, Charles LA, Jennette JC: Anti-neutrophil cytoplasmic autoantibodies induce neutrophils to degranulate and produce oxygen radicals in vitro. Proc Natl Acad Sci USA 1990, 87:4115-4119.

4. Savage CO, Pottinger BE, Gaskin G, Pusey CD, Pearson JD: Autoantibodies developing to myeloperoxidase and proteinase 3 in systemic vasculitis stimulate neutrophil cytotoxicity toward cultured endothelial cells. Am J Pathol 1992, 141:335-342.

5. Xiao H, Heeringa P, Liu Z, Huugen D, Hu P, Maeda N, Falk RJ, Jennette JC: The role of neutrophils in the induction of glomerulonephritis by antimyeloperoxidase antibodies. Am J Pathol 2005, 167:39-45.

6. Murphy PM: Neutrophil receptors for interleukin-8 and related CXC chemokines. Semin Hematol 1997, 34:311-318.

7. Pham CT: Neutrophil serine proteases: specific regulators of inflammation. Nat Rev Immunol 2006, 6:541-550.

8. Abdel-Salam B, Iking-Konert C, Schneider M, Andrassy K, Hansch GM: Autoantibodies to neutrophil cytoplasmic antigens (ANCA) do not bind to polymorphonuclear neutrophils in blood. Kidney Int 2004, 66:1009-1017.

9. Brachemi S, Mambole A, Fakhouri F, Mouthon L, Guillevin L, Lesavre P, Halbwachs-Mecarelli L: Increased membrane expression of proteinase 3 during neutrophil adhesion in the presence of anti proteinase 3 antibodies. J Am Soc Nephrol 2007, 18:2330-2339.

10. Reumaux D, Vossebeld PJ, Roos D, Verhoeven AJ: Effect of tumor necrosis factor-induced integrin activation on Fc gamma receptor II-mediated signal transduction: relevance for activation of neutrophils by antiproteinase 3 or anti-myeloperoxidase antibodies. Blood 1995, 86:3189-3195.

11. Van Rossum AP, Van Der Geld YM, Limburg PC, Kallenberg CG: Human anti-neutrophil cytoplasm autoantibodies to proteinase 3 (PR3-ANCA) bind to neutrophils. Kidney Int 2005, 68:537-541.

12. Jennette JC, Falk RJ, Andrassy K, Bacon PA, Churg J, Gross WL, Hagen EC, Hoffman GS, Hunder GG, Kallenberg CG: Nomenclature of systemic vasculitides. Proposal of an international consensus conference. Arthritis Rheum 1994, 37:187-192.

13. Satchell SC, Tasman CH, Singh A, Ni L, Geelen J, von Ruhland CJ, O'Hare MJ, Saleem MA, van den Heuvel LP, Mathieson PW: Conditionally immortalized human glomerular endothelial cells expressing fenestrations in response to VEGF. Kidney Int 2006, 69:1633-1640.

14. Hu N, Westra J, Huitema MG, Bijl M, Brouwer E, Stegeman CA, Heeringa P, Limburg PC, Kallenberg CG: Coexpression of CD177 and membrane proteinase 3 on neutrophils in antineutrophil cytoplasmic autoantibodyassociated systemic vasculitis: anti-proteinase 3-mediated neutrophil activation is independent of the role of CD177-expressing neutrophils. Arthritis Rheum 2009, 60:1548-1557.

15. Louis NA, Campbell E, Colgan SP: Model systems to investigate neutrophil adhesion and chemotaxis. In Neutrophil methods and protocols. Edited by: Quinn MT, Deleo FR, Bokoch GM. Totowa: Humana Press; 2007:257-272.

16. Zouki C, Beauchamp M, Baron C, Filep JG: Prevention of in vitro neutrophil adhesion to endothelial cells through shedding of L-selectin by C-reactive protein and peptides derived from C-reactive protein. $J$ Clin Invest 1997, 100:522-529.

17. Bertini R, Allegretti M, Bizzarri C, Moriconi A, Locati M, Zampella G, Cervellera MN, Di Cioccio V, Cesta MC, Galliera E, Martinez FO, Di Bitondo R, Troiani G, Sabbatini V, D'Anniballe G, Anacardio R, Cutrin JC, Cavalieri B, Mainiero F, Strippoli R, Villa P, Di Girolamo M, Martin F, Gentile M, 
Santoni A, Corda D, Poli G, Mantovani A, Ghezzi P, Colotta F: Noncompetitive allosteric inhibitors of the inflammatory chemokine receptors CXCR1 and CXCR2: prevention of reperfusion injury. Proc Nat Acad Sci USA 2004, 101:11791-11796.

18. Westra J, Brouwer E, van Roosmalen IA, Doornbos-van der Meer B, van Leeuwen MA, Posthumus MD, Kallenberg CG: Expression and regulation of HIF-1alpha in macrophages under inflammatory conditions; significant reduction of VEGF by CaMKII inhibitor. BMC Musculoskelet Disord 2010, $11: 61$.

19. Jonasdottir O, Petersen J, Bendtzen K: Tumour necrosis factor-alpha (TNF), lymphotoxin and TNF receptor levels in serum from patients with Wegener's granulomatosis. APMIS 2001, 109:781-786.

20. Ohlsson S, Wieslander J, Segelmark M: Circulating cytokine profile in antineutrophilic cytoplasmatic autoantibody-associated vasculitis: prediction of outcome? Mediators Inflamm 2004, 13:275-283.

21. Tesar V, Masek Z, Rychlik I, Merta M, Bartunkova J, Stejskalova A, Zabka J, Janatkova I, Fucikova T, Dostal C, Becvar R: Cytokines and adhesion molecules in renal vasculitis and lupus nephritis. Nephrol Dial Transplant 1998, 13:1662-1667.

22. Kumpers $P$, Hellpap J, David S, Horn R, Leitolf H, Haller H, Haubitz M: Circulating angiopoietin-2 is a marker and potential mediator of endothelial cell detachment in ANCA-associated vasculitis with renal involvement. Nephrol Dial Transplant 2009, 24:1845-1850.

23. Lemieux C, Maliba R, Favier J, Theoret JF, Merhi Y, Sirois MG: Angiopoietins can directly activate endothelial cells and neutrophils to promote proinflammatory responses. Blood 2005, 105:1523-1530.

24. Bauer S, Abdgawad M, Gunnarsson L, Segelmark M, Tapper H, Hellmark T: Proteinase 3 and CD177 are expressed on the plasma membrane of the same subset of neutrophils. J Leukoc Biol 2007, 81:458-464.

25. Rose JJ, Foley JF, Murphy PM, Venkatesan S: On the mechanism and significance of ligand-induced internalization of human neutrophil chemokine receptors CXCR1 and CXCR2. J Biol Chem 2004, 279:24372-24386.

26. van der Veen BS, Petersen AH, Belperio JA, Satchell SC, Mathieson PW Molema G, Heeringa P: Spatiotemporal expression of chemokines and chemokine receptors in experimental anti-myeloperoxidase antibodymediated glomerulonephritis. Clin Exp Immunol 2009, 158:143-153.

27. Godaly G, Hang L, Frendeus B, Svanborg C: Transepithelial neutrophi migration is CXCR1 dependent in vitro and is defective in IL-8 receptor knockout mice. J Immunol 2000, 165:5287-5294.

28. Mintz R, Garty BZ, Meshel T, Marcus N, Katanov C, Cohen-Hillel E, Ben Baruch A: Reduced expression of chemoattractant receptors by polymorphonuclear leukocytes in Hyper lgE Syndrome patients. Immunol Lett 2010, 130:97-106.

29. Olszyna DP, Florquin S, Sewnath M, Branger J, Speelman P, van Deventer SJ, Strieter RM, van der Poll T: CXC chemokine receptor 2 contributes to host defense in murine urinary tract infection. J Infect Dis 2001, 184:301-307.

30. Slocombe RF, Malark J, Ingersoll R, Derksen FJ, Robinson NE: Importance of neutrophils in the pathogenesis of acute pneumonic pasteurellosis in calves. Am J Vet Res 1985, 46:2253-2258.

31. Svensson M, Irjala H, Svanborg C, Godaly G: Effects of epithelial and neutrophil CXCR2 on innate immunity and resistance to kidney infection. Kidney Int 2008, 74:81-90.

32. Muller Kobold AC, Mesander G, Stegeman CA, Kallenberg CG, Tervaert JW: Are circulating neutrophils intravascularly activated in patients with antineutrophil cytoplasmic antibody (ANCA)-associated vasculitides? Clin Exp Immunol 1998, 114:491-499.

33. Casilli F, Bianchini A, Gloaguen I, Biordi L, Alesse E, Festuccia C, Cavalieri B, Strippoli R, Cervellera MN, Di Bitondo R, Ferretti E, Mainiero F, Bizzarri C, Colotta F, Bertini R: Inhibition of interleukin-8 (CXCL8/IL-8) responses by repertaxin, a new inhibitor of the chemokine receptors CXCR1 and CXCR2. Biochem Pharmacol 2005, 69:385-394.

34. Cohen-Hillel E, Yron I, Meshel T, Ben Baruch A: Interleukin 8 and cell migration to inflammatory sites: the regulation of focal adhesion kinase under conditions of migratory desensitization. Isr Med Assoc J 2007 9:579-583

35. Coelho FM, Pinho V, Amaral FA, Sachs D, Costa W, Rodrigues DH, Vieira AT, Silva TA, Souza DG, Bertini R, Teixeira AL, Teixeira MM: The chemokine receptors CXCR1/CXCR2 modulate antigen-induced arthritis by regulating adhesion of neutrophils to the synovial microvasculature. Arthritis Rheum 2008, 58:2329-2337.
36. Savage CO, Brooks CJ, Adu D, Richards G, Howie AJ: Cell adhesion molecule expression within human glomerular and kidney organ culture. J Pathol 1997, 181:111-115.

37. Lally F, Smith E, Filer A, Stone MA, Shaw JS, Nash GB, Buckley CD, Rainger GE: A novel mechanism of neutrophil recruitment in a coculture model of the rheumatoid synovium. Arthritis Rheum 2005, 52:3460-3469.

38. Hechtman DH, Cybulsky MI, Fuchs HJ, Baker JB, Gimbrone MA Jr: Intravascular IL-8. Inhibitor of polymorphonuclear leukocyte accumulation at sites of acute inflammation. J Immunol 1991 147:883-892

39. Simonet WS, Hughes TM, Nguyen HQ, Trebasky LD, Danilenko DM, Medlock ES: Long-term impaired neutrophil migration in mice overexpressing human interleukin-8. J Clin Invest 1994, 94:1310-1319.

40. Cockwell P, Brooks CJ, Adu D, Savage CO: Interleukin-8: A pathogenetic role in antineutrophil cytoplasmic autoantibody-associated glomerulonephritis. Kidney Int 1999, 55:852-863.

41. Mayet W, Schwarting A, Barreiros AP, Schlaak J, Neurath M: Anti-PR-3 antibodies induce endothelial IL-8 release. Eur J Clin Invest 1999, 29:973-979.

42. Strieter RM, Kunkel SL, Showell HJ, Remick DG, Phan SH, Ward PA, Marks RM: Endothelial cell gene expression of a neutrophil chemotactic factor by TNF-alpha, LPS, and IL-1 beta. Science 1989, 243:1467-1469.

43. Abdulahad WH, Stegeman CA, Limburg PC, Kallenberg CG: Skewed distribution of Th17 lymphocytes in patients with Wegener's granulomatosis in remission. Arthritis Rheum 2008, 58:2196-2205.

44. Khandaker MH, Mitchell G, Xu L, Andrews JD, Singh R, Leung H, Madrenas J, Ferguson SS, Feldman RD, Kelvin DJ: Metalloproteinases are involved in lipopolysaccharide- and tumor necrosis factor-alpha-mediated regulation of CXCR1 and CXCR2 chemokine receptor expression. Blood 1999, 93:2173-2185

45. Chan AT, Flossmann O, Mukhtyar C, Jayne DR, Luqmani RA: The role of biologic therapies in the management of systemic vasculitis. Autoimmun Rev 2006, 5:273-278.

46. Huugen D, Xiao H, van Esch A, Falk RJ, Peutz-Kootstra CJ, Buurman WA, Tervaert JW, Jennette JC, Heeringa P: Aggravation of antimyeloperoxidase antibody-induced glomerulonephritis by bacterial lipopolysaccharide: role of tumor necrosis factor-alpha. Am J Pathol 2005, 167:47-58.

47. Noronha IL, Kruger C, Andrassy K, Ritz E, Waldherr R: In situ production of TNF-alpha, IL-1 beta and IL-2R in ANCA-positive glomerulonephritis. Kidney Int 1993, 43:682-692.

\section{doi:10.1186/ar3534}

Cite this article as: Hu et al:: Decreased CXCR1 and CXCR2 expression on neutrophils in anti-neutrophil cytoplasmic autoantibody-associated vasculitides potentially increases neutrophil adhesion and impairs migration. Arthritis Research \& Therapy 2011 13:R201.

\section{Submit your next manuscript to BioMed Central and take full advantage of:}

- Convenient online submission

- Thorough peer review

- No space constraints or color figure charges

- Immediate publication on acceptance

- Inclusion in PubMed, CAS, Scopus and Google Scholar

- Research which is freely available for redistribution

Submit your manuscript at www.biomedcentral.com/submit
C Biomed Central 